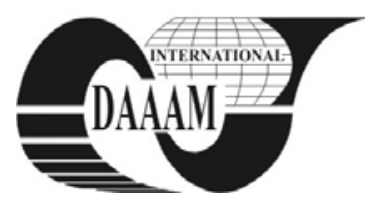

\title{
APERIODIC PRINCIPLE FOR AUTOMATIC CONTROLLER TUNING
}

\author{
KORBEL, J[iri] \& PROKOP, R[oman]
}

\begin{abstract}
This paper presents simple principle for aperiodic tuning of SISO controllers used in autotuning. Autotuners represent a combination of relay feedback identification and some control design method. In this contribution, models with up to three parameters are estimated by means of a single asymmetrical relay experiment. Then a stable low order transfer function is identified. Further, the controller is derived from general solutions of Diophantine equations in the ring of proper and stable rational functions. A first order identification yields a PI-like controllers while a second order generates PID ones. The analytical simple rule is derived for aperiodic control response and the scalar tuning parameter $m>0$ is then tuned according to identified time constant of an approximated transfer function.
\end{abstract}

Key words: autotuning, algebraic design, relay, pole-placement

\section{INTRODUCTION}

Industrial processes are usually controlled by PID controllers, (Yu, 1999) refers that more than $97 \%$ of control loops are of this type and most of them are actually under PI control. The advantages of PID controllers can be seen in a simple structure, understandable principle and control capabilities. It is widely known that PID controllers are quite resistant to changes in the controlled process without meaningful deterioration of the loop behavior. For many years, the Ziegler - Nichols tuning rule has been applied for controller tuning. However, there are many limitations, drawbacks and infirmities in the behavior. A solution for qualified choice of controller parameters can be seen in more sophisticated, proper and automatic tuning of PID controllers.

The development of various autotuning principles was started by (Åström \& Hägglund, 1984) in the year 1984. During the period of more than two decades, many studies have been reported to extend and improve autotuners principles; see e.g. (Åström \& Hägglund, 1995; Garcia \& Castelo, 2000). The extension in relay utilization was performed in (Pecharromán \& Pagola, 2000; Thyagarajan \& Yu, 2002) by an asymmetry and hysteresis of a relay. Experiments with asymmetrical and deadzone relay feedback are reported in (Vítečková \& Víteček, 2004). Nowadays, almost all commercial industrial PID controllers provide the feature of autotuning.

In this paper, a new combination for autotunig method with an aperiodic control rule is proposed and developed. The basic autotuning principle combines an asymmetrical relay identification experiment and a control design performed in the ring of proper and stable rational functions $R_{P S}$. The pole placement problem in $R_{P S}$ ring is formulated through a Diophantine equation and the pole is analytically tuned according to aperiodic response of the closed loop. Naturally, there exist many principles of control design synthesis which can be used for autotuning principles, e.g. (O’Dwyer, 2003). They can be considered as alternative approach and only practical application test their abilities.

\section{CONTROLLERS DESIGN}

The control design is based on the fractional approach; see e.g. (Vidyasagar, 1987; Kučera, 1993). Any transfer function G(s) of a (continuous-time) linear system is expressed as a ratio of two elements of $R_{P S}$. The set $R_{P S}$ means the ring of (Hurwitz) stable and proper rational functions. Then all transfer functions can be expressed by the ratio:

$$
\begin{gathered}
G(s)=\frac{b(s)}{a(s)}=\frac{\frac{b(s)}{(s+m)^{n}}}{\frac{a(s)}{(s+m)^{n}}}=\frac{B(s)}{A(s)} \\
n=\max (\operatorname{deg}(a), \operatorname{deg}(b)), \quad m>0
\end{gathered}
$$

All feedback stabilizing controllers are given by a general solution of the Diophantine equation:

$$
A P+B Q=1
$$

which can be expressed with $Z$ free in $\mathrm{R}_{\mathrm{PS}}$ :

$$
\frac{Q}{P}=\frac{Q_{0}-A Z}{P_{0}+B Z}
$$

Diophantine equation (3) for the first order systems without the time delay can be easily transformed into polynomial equation:

$$
\frac{(T s+1)}{s+m} p_{0}+\frac{K}{s+m} q_{0}=1
$$

with general solution:

$$
\begin{aligned}
& P=\frac{1}{T}+\frac{K}{s+m} \cdot Z \\
& Q=\frac{T m-1}{T K}-\frac{T s+1}{s+m} \cdot Z
\end{aligned}
$$

where $Z$ is free in $\mathrm{R}_{\mathrm{PS}}$. Asymptotic tracking is achieved by:

$$
Z=-\frac{m}{T K}
$$

and the resulting PI controller is in the form:

$$
C(s)=\frac{Q}{P}=\frac{q_{1} s+q_{0}}{s}
$$

where parameters $q_{1}$ a $q_{0}$ are given by:

$$
q_{1}=\frac{2 T m-1}{K} \quad q_{0}=\frac{T m^{2}}{K}
$$




\section{APERIODIC TUNING OF CONTROLLERS}

A simple and attractive choice for the tuning parameter $m>0$ can be easily obtained analytically. In the $\mathrm{R}_{\mathrm{PS}}$ expression, the closed-loop transfer function $K_{w y}$ is for first order system and PI controller (8) given in a very simple form:

$$
K_{w y}=\frac{B Q}{A P+B Q}=B Q=\frac{(2 T m-1) s+T m^{2}}{(s+m)^{2}}
$$

The step response of (10) can be expressed by Laplace transform:

$$
\begin{aligned}
h(t) & =L^{-1}\left\{\frac{K_{w y}}{s}\right\}=L^{-1}\left\{\frac{k_{1} s+k_{0}}{s(s+m)^{2}}\right\}= \\
& =L^{-1}\left\{\frac{A}{s}+\frac{B}{(s+m)}+\frac{C}{(s+m)^{2}}\right\},
\end{aligned}
$$

where $A, B, C$ are calculated by comparing appropriate fractions in (11) and $k_{1}=2 m T-1, k_{0}=T m^{2}$. The step response $h(t)$ in time domain is then:

$$
h(t)=A+B e^{-m t}+C t e^{-m t}
$$

The overshoot or undershoot of this response is characterized by the first derivative condition:

$$
h^{\prime}(t)=-m B e^{-m t}+C\left(e^{-m t}-t m e^{-m t}\right)=0
$$

From (13) time of the extreme of response $h(t)$ is then easily calculated by the relation:

$$
t_{e}=\frac{C-m B}{m C}=\frac{1}{m}-\frac{B}{C}
$$

Since the aperiodic response means that the extreme does not exist for positive $t_{e}$, it implies $t_{e}<0$ and after all substitutions of $A, B, C, k_{1}, k_{0}$ relation (14) takes the simple form:

$$
1<m \frac{B}{C}=\frac{1}{\frac{1}{T m}-1}
$$

The denominator of (15) must be positive and less than 1 and $m>0$ which implies the inequality:

$$
\frac{1}{2 T}<m<\frac{1}{T}
$$

Any positive parameter $m$ from (16) ensures aperiodic response. It is a question for further investigation and simulation what choice from interval is the best.

\section{SIMULATION EXAMPLE}

A second order system G(s) without a time delay was estimated by a first order model in the above mentioned relay experiment. Both transfer functions have the form:

$$
G(s)=\frac{3}{(2 s+1)^{2}} \quad \tilde{G}(s)=\frac{3.02}{3.89 s+1} \cdot e^{-1.15 s}
$$

The relay experiments enable to estimate the original system by the first or second order transfer functions. The PI controller generated from the approximated system was designed by equations (8) and (9) for three values of tuning parameters $m>0$ with respect the interval (16). The responses are shown in Fig. 1.

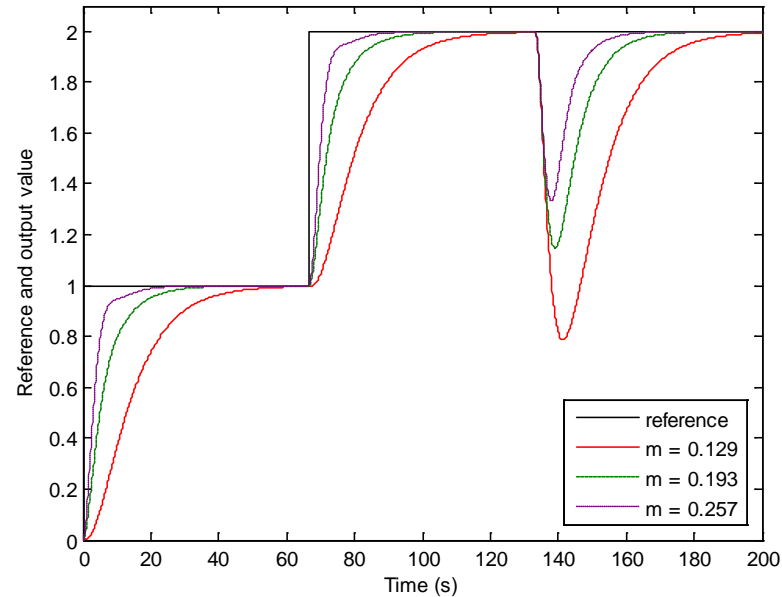

Fig. 1 Control responses of the system

\section{CONCLUSION}

This contribution gives a new combination of relay feedback identification and a control design method. The estimation of a low order transfer function parameters is performed from asymmetric limit cycle data. The control synthesis is carried out through the solution of a linear Diophantine equation. This approach brings a scalar tuning parameter which can be adjusted by various strategies. A first order estimated model generates PI-like controllers. The aperiodic tuning through the parameter $m>0$ is proposed by the analytic derivation.

\section{ACKNOWLEDGEMENTS}

This work was supported by the Ministry of Education, Youth and Sports of the Czech Republic under the Research Plan No. MSM 7088352102 and by the European Regional Development Fund under the project CEBIA-Tech No. CZ.11.05./2.1.00 /03.0089.

\section{REFERENCES}

Åström, K.J. \& Hägglund, T. (1984). Automatic tuning of simple regulators with specification on phase and amplitude margins. Automatica, Vol.20, pp.645-651

Åström, K.J. \& Hägglund, T. (1995). PID Controllers: Theory, Design and Tuning. Research Triangle Park, NC: Instrumental Society of America

Garcia, R.F. \& Castelo, F.J.P. (2000). A complement to autotuning methods on PID controllers, In: Preprints of IFAC Workshop PID'00, pp. 101-104

Kučera, V. (1993). Diophantine equations in control - A survey, Automatica, Vol. 29, No. 6, pp. 1361-75

O'Dwyer, A. (2003). Handbook of PI and PID controller tuning rules. London: Imperial College Press

Pecharromán, R.R. \& Pagola, F.L. (2000). Control design for PID controllers auto-tuning based on improved identification, In: Preprints of IFAC Workshop PID'00, pp. 89-94

Thyagarajan, T. \& Yu, Ch.Ch. (2002). Improved autotuning using shape factor from relay feedback, In: Preprints of IFAC World Congress

Vidyasagar, M. (1987). Control system synthesis: a factorization approach. MIT Press, Cambridge, M.A

Vítečková, M. \& Víteček, A. (2004). Experimentální identifikace metodou relé (in Czech), In: Automatizácia a informatizácia

Yu, Ch.Ch. (1999). Autotuning of PID Controllers. Springer, London 